\title{
EFECTO DE LA ADICIÓN DE POLÍMEROS DE CADENA CORTA SOBRE LA ESTRUCTURA QUÍMICA, PROPIEDADES MECÁNICAS, TÉRMICAS Y BIOLÓGICAS DE POLIURETANOS SINTETIZADOS CON DIISOCIANATOS ALIFÁTICOS Y ACEITE DE HIGUERILLA
}

\author{
Yomaira L. Uscategui ${ }^{\mathrm{a}}$, Luis E. Díaz ${ }^{\mathrm{a},(\mathbb{1}}$ y Manuel F. Valero ${ }^{\mathrm{a}, *,(])}$ \\ ${ }^{a}$ Facultad de Ingeniería, Universidad de La Sabana, Campus Universitario del Puente del Común, Autopista Norte de Bogotá, D.C. \\ Chía, Cundinamarca, Colombia
}

Recebido em 02/07/2020; aceito em 11/08/2020; publicado na web em 21/09/2020

\begin{abstract}
EFFECT OF THE ADDITION OF SHORT CHAIN POLYMERS ON THE CHEMICAL STRUCTURE, MECHANICAL, THERMAL AND BIOLOGICAL PROPERTIES OF POLYURETHANES SYNTHESIZED WITH ALIPHATIC DIISOCYANATES AND CASTOR OIL. The objective of this research was to synthesize biocompatible polyurethanes, with suitable mechanical properties to be used as biomaterials. Polyurethanes were synthesized with polyols derived from castor oil and isophorone diisocyanate (IPDI), hexamethylene diisocyanate and 4,4'-methylenebis (cyclohexyl isocyanate), with the addition of polycaprolactone/chitosan (PCL/Ch). The materials were evaluated for their mechanical properties by determining the stress-strain curves, thermogravimetric analysis, differential scanning calorimetry analysis and contact angle. The biological response of the materials was evaluated by measuring in vitro cell viability on the L-929 mouse fibroblast cell line. The mechanical properties of polyurethanes vary according to the type of polyol and diisocyanate used. The best results were obtained with transesterificationmodified polyols with cycloaliphatic diisocyanates. The best results were found for polyurethane synthesized with IPDI and the modified polyol (P.3): $17 \mathrm{MPa}$ of maximum stress at break, $0.937 \mathrm{MPa}$ of Young's modulus, elongation greater than $250 \%$. Polyurethanes do not have toxic effects and cell viability values exceed $70 \%$ on the L-929 fibroblast line. The addition of PCL/Ch improves the cell viability of polyurethanes. The results indicate that the polyurethanes synthesized with polyols derived from castor oil can be used in the design of biomaterials due to the mechanical and biological properties obtained.
\end{abstract}

Keywords: polyurethanes; aliphatic diisocyanates; polycaprolactone; chitosan; biomaterials.

\section{INTRODUCCIÓN}

Los poliuretanos (PUs) son polímeros sintéticos, compuestos por unidades orgánicas unidas por enlaces uretano. ${ }^{1}$ Son polímeros segmentados bastante versátiles ya que se caracterizan por presentar buenas propiedades mecánicas, físicas y químicas junto con buenas propiedades biocompatibles. ${ }^{1,2}$ Esta versatilidad permite que los PUs tengan aplicaciones en diversas áreas a nivel industrial como por ejemplo los neumáticos, materiales para la construcción, automóviles, calzado, etc. ${ }^{1,3}$ Adicionalmente, la variabilidad de los PUs permite tener aplicaciones en el campo biomédico como dispositivos cardiovasculares, catéteres vasculares, sistemas de administración de fármacos, entre otros. ${ }^{1,4}$

La diversidad de los PUs está dada por los diferentes monómeros empleados en el proceso de síntesis incluyendo los aditivos utilizados. Los PUs se conocen como copolímeros de bloque (segmentos alternos blandos y duros) que generalmente se obtiene por la reacción entre un diol o poliol, que le confiere el segmento blando, y un isocianato que junto con un extensor de cadena le confiere el segmento duro. ${ }^{5}$ Los isocianatos se caracterizan por presentar una elevada reactividad y versatilidad debido a que poseen el grupo funcional NCO. ${ }^{6}$ Generalmente se utilizan diisocianatos alifáticos y aromáticos para la síntesis de PUs los cuales tienen una fuerte influencia sobre el proceso de polimerización y la estructura del polímero final, ${ }^{1,4}$ lo que influye directamente sobre las propiedades mecánicas y biológicas del material. ${ }^{7}$

Los polímeros sintéticos tradicionales utilizan materias primas derivadas de combustibles fósiles lo que ha generado daños irreversibles a los ecosistemas naturales. ${ }^{8,9}$ Es por esto que existe

*e-mail: manuel.valero@unisabana.edu.co una necesidad de utilizar materias primas biodegradables amigables con el ecosistema natural. Una opción consiste en utilizar polioles para la síntesis de PUs derivados de fuentes vegetales. Es así como los aceites vegetales actualmente presentan un enorme potencial como monómeros en la síntesis de PUs y poli (éter uretanos), ${ }^{10}$ como materiales renovables en aras de buscar un desarrollo sostenible. ${ }^{6,9}$ Los triglicéridos de diferentes aceites vegetales se han empleado en la síntesis de PUs como el aceite de neem (Azadirachta indica), aceite de jatropha (Jatropha curcas), entre otros, por su elevado peso molecular. ${ }^{6,10,11}$ Otro aceite promisorio y que se emplea como poliol en la síntesis de PUs es el aceite de higuerilla que se caracteriza por presentar algunas ventajas frente a otros aceites vegetales, como la posibilidad de emplearlo de forma directa en el proceso de síntesis gracias a la elevada composición del triglicérido del ácido ricinoléico y la presencia de grupos hidroxilos en la estructura química del aceite. ${ }^{12,13}$ Adicional a esto, la presencia de dobles enlaces éster en el triglicérido ofrece varias rutas para modificar la estructura por medio de la adición de grupos hidroxilo necesarios para la síntesis de PUs. ${ }^{14}$ Los PUs que utilizan aceite de higuerilla en la síntesis con diferentes diisocianatos se presentan como alternativas renovables, según el concepto de desarrollo sostenible.

Aunque los PUs son ampliamente utilizados como biomateriales tanto por su comportamiento mecánico como biocompatible, aún se realizan investigaciones para mejorar propiedades como la biocompatibilidad, actividad antimicrobiana o hemocompatibilidad. ${ }^{8}$ Esto se puede lograr por medio del uso de materias primas como aditivos en la síntesis de PUs que a su vez sean amigables con el medio ambiente, como son los polímeros de cadena corta biodegradables. Polímeros naturales como el quitosano $(\mathrm{Ch})$ o polímeros sintéticos como la policaprolactona (PCL) han recibido atención en los procesos actuales. ${ }^{8}$ Polisacáridos como el Ch ofrece algunos beneficios en la 
síntesis de polímeros por conseguirse de forma natural, presentar propiedades biocompatibles, no ser tóxico, biodegradable, entre otros. ${ }^{15,16}$ De otro lado, la PCL se caracteriza por ser biodegradable, biocompatible, no tóxico, entre otras propiedades. ${ }^{17}$ Todas estas propiedades, especialmente la biocompatibilidad y bioactividad, convierten tanto a la PCL como al Ch en candidatos para la síntesis de diversos materiales para el uso en el campo biomédico. ${ }^{18}$

Es por lo anterior que se han desarrollado investigaciones previas en nuestro grupo de investigación relacionadas con el uso de aceite de higuerilla como poliol y diisocianato de isoforona en la síntesis de PUs para obtener materiales candidatos en aplicaciones biomédicas. Para alterar las propiedades de las matrices de los PUs se varió la funcionalidad del poliol por medio de la modificación química del aceite de higuerilla y se sintetizaron PUs con IPDI obteniendo materiales con un incremento en las propiedades mecánicas, mayor estabilidad térmica, materiales con propiedades antibacterianas in vitro y no presentaban toxicidad frente a líneas celulares L-929 y NIH/3T3, lo cual indicaría un posible uso de los PUs como biomateriales. ${ }^{12}$ También se evaluaron materiales sintetizados con polioles modificados del aceite de higuerilla con IPDI y la adición de PCL para mejorar propiedades biológicas y se encontró que los materiales son aptos para el diseño de biomateriales para tejidos bandos o suturas biomédicas. ${ }^{19}$ PUs sintetizados con aceite de higuerilla, IPDI y nanocristales de celulosa presentaron mejoras en propiedades térmicas y mecánicas de los materiales, incremento en el carácter hidrofílico, viabilidad celular superior al $70 \%$ sobre la línea celular L-929, con lo que se proponen estos materiales como posibles candidatos para el desarrollo de biomateriales activos. ${ }^{20}$ Ahora lo que se quiere evaluar es el efecto de utilizar otros diisocianatos alifáticos sobre las propiedades de los PUs junto con la adición de la mezcla de PCL/Ch en aras de buscar materiales aptos para el diseño de biomateriales. Lo anterior es teniendo en cuenta que la síntesis de PUs con diisocianatos aromáticos no son biológicamente activas por la formación de residuos altamente tóxicos durante la degradación (como la formación de diaminas aromáticas), limitando así el uso de los estos materiales sintéticos en aplicaciones biotecnológicas. ${ }^{10}$ Es así como en esta investigación se utilizaran tres diisocianatos alifáticos, uno lineal (HDI), otro con un anillo (IPDI) y el otro con dos anillos (HMDI), para evitar efectos secundarios de los materiales sobre los tejidos vivos.

Por lo tanto, el objetivo de este artículo fue sintetizar materiales tipo PUs con tres polioles derivados del aceite de higuerilla (Ricinus communis) y tres diisocianatos, diisocianato de isoforona (IPDI), diisocianato de hexametileno (HDI) y 4,4'-metilenbis(isocianato de ciclohexilo) (HMDI), con adición de PCL (15\% p/p) y Ch (3\% p/p), para evaluar las propiedades fisicoquímicas, mecánicas y térmicas de los materiales en función de la estructura química de los monómeros empleados para la síntesis junto con la evaluación de la viabilidad celular de células de fibroblastos de ratón L-929 en contacto con los materiales. Al poder relacionar la estructura de los monómeros empleados con las propiedades de los PUs sintetizados, se podrá determinar si los PUs pueden ser candidatos en aplicaciones biomédicas.

\section{EXPERIMENTAL}

\section{Reactivos}

Aceite de higuerilla (Ricinus communis) adquirido en Químicos Campota y Cía, Ltda., Colombia. Diisocianato de isoforona (IPDI), diisocianato de hexametileno (HDI), 4,4'-metilenbis(isocianato de ciclohexilo) (HMDI), policaprolactona diol (PCL) con peso molecular promedio de $2000 \mathrm{~g} \mathrm{~mol}^{-1}$ y quitosano (Ch) de bajo peso molecular con un porcentaje de deacetilación entre $75-85 \%$ eran marca Sigma-Aldrich Chemical Co. (St. Louis, MO, USA). Pentaeritritol marca Merck \& Co., Inc. (Alemania). Buffer fosfato salino (PBS: 0,01 M KH2PO4-NaHPO4 con 0,8\% NaCl, pH 7,4). MTT (3-[4,5-dimethylthiazol-2-yl]-2,5-diphenyl-2H-tetrazolium bromide), tripsina $2.5 \%$ (10X), Penicilin-Streptomycin (10.000 Und de penicilina y $10.000 \mu \mathrm{g}$ de estreptomicina por mililitro), D-MEM (Dulbecco's Modified Eagle Medium) (1X), fueron marca Gibco/ Invitrogen, Paisley, UK. Suero fetal bovino (FBS) marca Eurobio (Les Ulis, France). Caldo tripticasa soya (TSB) y Agar tripticasa soya (TSA) marca Scharlau Co (España). Fibroblastos de tejido conectivo subcutáneo de ratón L-929 (ATCC® CCL-1).

\section{Síntesis de PUs}

Para la síntesis de los PUs se utilizó un diseño factorial completo para estudiar el efecto de los factores sobre las variables de respuesta. Se utilizaron 3 factores, 2 variables categóricas con 3 niveles cada uno, y una variable numérica con 2 niveles, para un total de 18 láminas sintetizadas. Las variables categóricas corresponden a tres tipos de polioles y tres diisocianatos, y la variable numérica corresponde a la adición de policaprolactona (PCL) al 15\% p/p con quitosano (Ch) al $3 \% \mathrm{p} / \mathrm{p}$.

Los tres polioles se obtuvieron del aceite de higuerilla (P1, P2 y P3) por medio de modificación química por transesterificación con pentaeritritol (sin modificar, con $1,32 \%$ y con $2,64 \%$ mol de pentaeritritol $/ \mathrm{mol}$ de aceite de higuerilla, respectivamente) a $210{ }^{\circ} \mathrm{C}$, con óxido de plomo $(0,5 \%)$ como catalizador durante $2 \mathrm{~h}^{21}$

Los tres diisocianatos utilizados fueron: diisocianato de hexametileno (HDI), diisocianato de isoforona (IPDI) y 4,4'-metilenbis(isocianato de ciclohexilo) (HMDI).

Los PUs fueron sintetizados por el método del pre-polímero en una relación constante $\mathrm{NCO} / \mathrm{OH}(1: 1) .{ }^{21} \mathrm{El}$ poliol se llevó a $60^{\circ} \mathrm{C}$ y se adicionó el diisocianato a $300 \mathrm{rpm}$ por $5 \mathrm{~min}$. Luego se adicionó PCL $(15 \% \mathrm{p} / \mathrm{p}$ con respecto a la masa del poliol del aceite) y Ch $(3 \% \mathrm{p} / \mathrm{p}$ con respecto a la masa del poliol del aceite) a $300 \mathrm{rpm}, 60{ }^{\circ} \mathrm{C}$ por $5 \mathrm{~min}$. Los PUs fueron vertidos en moldes de $15 \mathrm{~cm} \times 9 \mathrm{~cm} \times 0,3 \mathrm{~cm}$ (largo $\times$ ancho $\times$ alto) y se llevaron a $110{ }^{\circ} \mathrm{C}$ durante $12 \mathrm{~h} .{ }^{21}$

\section{Caracterización de PUs}

Espectroscopia de infrarrojo por transformada de Fourier (FTIR)

Para determinar la estructura química de los PUs se utilizó un espectrómetro ATR-FTIR (Bruker ALPHA), en un intervalo de 400 y $4000 \mathrm{~cm}^{-1}$, con un promedio de 24 escaneos a una resolución espectral de $4 \mathrm{~cm}^{-1}$. $22,23^{2}$

\section{Determinación de la densidad}

La densidad de los materiales se determinó utilizando la técnica del empuje de Arquímedes con una balanza Mettler AX 205 (MettlerToledo Inc.) con una sensibilidad de $0.01 \mathrm{mg}$ y con un kit para determinar densidad Mettler ME 33360. Se utilizaron muestras secas de $5 \mathrm{~mm} \times 5 \mathrm{~mm} \times 3 \mathrm{~mm}$ para calcular la densidad por triplicado. Los PUs se pesaron en el aire $\left(\mathrm{m}_{\text {aire }}\right)$ y luego se sumergieron en n-octano con una densidad conocida $\left(\rho_{\text {n-octano }}\right)$ y se obtuvo el peso en inmersión $\left(\mathrm{m}_{\mathrm{n} \text {-octano }}\right)$ a $20^{\circ} \mathrm{C}$. La densidad se calculó por medio de la siguiente ecuación $1:^{24}$

$$
\text { Densidad de } P U S=\frac{m_{\text {aire }} \rho_{n-\text { octano }}}{m_{\text {aire }}-m_{n-\text { octano }}}
$$

Propiedades mecánicas

Se utilizó una máquina de tracción universal EZ-LX (Shimadzu, Japón) para determinar el esfuerzo máximo, el porcentaje de 
elongación y el módulo de Young de los PUs. Las condiciones de trabajo fueron: una celda de carga de $5 \mathrm{kN}$, una velocidad de desplazamiento de $25 \mathrm{~mm} \mathrm{~min}^{-1}$. Para este ensayo se probaron tres muestras de $40 \pm 0.02 \mathrm{~mm} \times 6 \pm 0.02 \mathrm{~mm} \times 3 \pm 0.02 \mathrm{~mm}$ (largo $\times$ ancho $\times$ espesor).

\section{Propiedades térmicas}

Para el análisis termogravimétrico se utilizó un analizador termogravimétrico acoplado a calorimetría diferencial de barrido modelo TGA/DCS1 (Mettler Toledo, USA). Se utilizó una velocidad de calentamiento de $25^{\circ} \mathrm{C} \mathrm{min}{ }^{-1}$, temperatura de $25-600{ }^{\circ} \mathrm{C}$, atmósfera de nitrógeno y muestras de $15 \pm 2 \mathrm{mg}$. Se realizó una calorimetría diferencial de barrido (DSC) para determinar la temperatura de transición vítrea por medio de un analizador DSC modelo DSC 3+ (Mettler Toledo, USA). Se utilizó una temperatura desde $-70^{\circ} \mathrm{C}$ hasta $150{ }^{\circ} \mathrm{C}$, atmósfera de nitrógeno con $20 \mathrm{~mL} \mathrm{~min}^{-1}$ de flujo, velocidad de calentamiento de $20^{\circ} \mathrm{C}$ min $^{-1}$ y un tamaño de muestra de $10 \pm 2 \mathrm{mg}$.

\section{Ángulo de contacto}

Se utilizó el sistema Drop Shape Analysis - DSA (GH11, Krüss, Alemania) para determinar el ángulo de contacto. Se utilizó el método sessile drop con $10 \mu \mathrm{L}$ de agua destilada a $20^{\circ} \mathrm{C}$. Se realizaron 10 mediciones de cada material.

\section{Evaluación biológica de PUs}

\section{Ensayo in vitro de viabilidad celular}

Para determinar la viabilidad celular se utilizó el método MTT definido por ISO/CD 10993-5 con fibroblastos de ratón L-929 cultivados en D-MEM, suplementado con suero bovino fetal (10\%) y penicilina-streptomicina (1\%) en frascos de cultivo celular T75. Se cultivaron a $37{ }^{\circ} \mathrm{C}$ y $5 \%$ de $\mathrm{CO}_{2}$. Se realizó cambio de medio a las células cada $48 \mathrm{~h} .{ }^{25} \mathrm{~A}$ una confluencia del $100 \%$, las células se tripsinizaron (tripsina EDTA) para análisis de viabilidad.

Los fibroblastos se sembraron en platos de 96 pozos a una concentración de $4.0 \mathrm{E}+04$ células por pozo con medio suplementado a $37{ }^{\circ} \mathrm{C}$ y $5 \% \mathrm{CO}_{2}$. Se adicionaron cilindros de PUs de $3 \times 2 \mathrm{~mm}^{2}$ (diámetro $\times$ largo) previamente esterilizados con luz UV $(260 \mathrm{~nm})$ durante $30 \mathrm{~min}$ por cada lado, ${ }^{26}$ y se agregaron $100 \mu \mathrm{L}$ de medio suplementado durante $24 \mathrm{~h}$ a $37^{\circ} \mathrm{C}$ y $5 \% \mathrm{CO}_{2}$. Se adicionaron $100 \mu \mathrm{L}$ de la solución de MTT (12 $\mathrm{mmol} \mathrm{L}^{-1}$ en PBS) previa remoción del sobrenadante y PUs por $4 \mathrm{~h}$ a $37^{\circ} \mathrm{C}$. Luego se retiró la solución de MTT y se adicionaron $100 \mu \mathrm{L}$ de dimetilsulfoxido por 15 min a $37^{\circ} \mathrm{C}$. La viabilidad celular se determinó por medio de la lectura de la densidad óptica a $50 \mathrm{~nm}$ utilizando un lector de placas (Bio-Tek ELx800 Microplate Reader). Se utilizó un control positivo y uno negativo (polipropileno (PP) y doxorrubicina (Doxo), respectivamente). Todos los ensayos se realizaron por triplicado. Por medio de la ecuación 2 se calculó el porcentaje de viabilidad celular:

$$
\% \text { de viabilidad }=\frac{\left(A b s_{\text {muestra }}\right)}{A b s_{\text {control }}} * 100
$$

$\mathrm{Abs}_{\text {muestra }}$ son los fibroblastos luego del tratamiento con los materiales, y $\mathrm{Abs}_{\text {control }}$ corresponde a fibroblastos sin tratamiento.

\section{Análisis estadístico}

Todos los resultados se presentan como el promedio \pm desviación estándar (SD). Se presentan análisis de varianza (ANOVA) junto con las diferencias significativas determinadas para $\mathrm{p}<0.05$. Se utilizó la prueba t-Student del Software SPSS Statistics 23 para la comparación entre muestras.

\section{RESULTADOS Y DISCUSIÓN}

\section{Síntesis de PUs}

En esta investigación se utilizaron tres polioles y tres diisocianatos. Los monómeros empleados en la síntesis de los PUs corresponden a polioles derivados del aceite de higuerilla y diisocianatos alifáticos como se observa en la Figura 1.

Para obtener un incremento en la funcionalidad de los polioles derivados del aceite de higuerilla se utilizó un proceso de transesterificación como se observa en la Figura 1(a) donde se modificó el poliol por medio de la reacción con pentaeritritol. Se evaluó el índice hidroxilo de los polioles que corresponde a un valor numérico que representa la cantidad de grupos hidroxilo presente en cada poliol luego de la modificación química. ${ }^{14}$ Por lo tanto a los tres poliles derivados del aceite de higuerilla se les determinó el índice hidroxilo por medio de la norma ASTM D1639-90. Los valores obtenidos fueron 160, 191 y 236 mg KOH g${ }^{-1}$ de aceite de higuerilla, para P.1, P.2 y P.3, respectivamente. En la Figura 1(b) se presenta la estructura de los diisocianatos alifáticos empleados en la reacción de los PUs.

\section{Caracterización de PUs}

Espectroscopia de infrarrojo por transformada de Fourier (FTIR)

En la Figura 2 se presentan los espectros infrarrojo de los PUs sintetizados según el tipo de poliol y el diisocianato utilizado.

La estructura química de los PUs se confirmó por medio de las bandas de absorción infrarroja de la siguiente forma: alrededor de los $3330 \mathrm{~cm}^{-1}$ se observaron las bandas características a vibraciones de estiramiento de los enlaces - N-H característicos de los enlaces uretano. Cerca de los $2923 \mathrm{~cm}^{-1}$ se observa el estiramiento del grupo metil. Alrededor de los $2855 \mathrm{~cm}^{-1}$ se encuentra el estiramiento simétrico del enlace C-H. Cerca de $\operatorname{los} 1700 \mathrm{~cm}^{-1}$ se observa una banda característica de las vibraciones de estiramiento del enlace $\mathrm{C}=\mathrm{O}$ de los enlaces uretano. ${ }^{4} \mathrm{~A}$ los $1250 \mathrm{~cm}^{-1}$ se observa el estiramiento del enlace $\mathrm{C}-\mathrm{N}$, y cerca de los $1140 \mathrm{~cm}^{-1}$ se presentan las vibraciones de estiramiento del enlace C-O..$^{23,27}$ Con los resultados anteriores se puede inferir que las matrices sintetizadas corresponden a PUs ya que el nuevo producto sintetizado tiene un grupo uretano (NHCOO). ${ }^{10}$ De igual forma, se observa que los materiales que tienen adición de PCL/Ch no presentan diferencias significativas respecto de los materiales sin aditivos. Así como tampoco se presentan diferencias con los polioles utilizados o con el tipo de diisocianato con lo que se puede inferir que el material es un PU independiente del monómero empleado en la síntesis.

\section{Determinación de densidad}

La densidad de todos los materiales sintetizados se presenta en la Figura 3.

Según se observa en la Figura 3, la densidad de los PUs depende del tipo de poliol empleado y del tipo de diisocianato utilizado en la síntesis. A medida que incrementa la densidad de entrecruzamiento, se incrementa la densidad del material polimérico resultante de forma significativa, aunque la diferencia entre el valor más alto y el más bajo es inferior al 5\%, de acuerdo con lo que se observa para cada poliol. La estructura del diisocianato también afecta la densidad de los materiales ya que al parecer los diisocianatos que tienen estructuras cíclicas incrementan la densidad de los PUs. De otro lado, la adición de PCL/Ch tiene una tendencia a incrementar la densidad de los PUs posiblemente por el efecto de relleno que generan en los materiales. Lo anterior está de acuerdo con los resultados de Conejero-García y col. quienes sintetizaron poliglicerol sebacato con distinto grado de entrecruzamiento como material para diversas aplicaciones en el 
(a)<smiles>[R]C(=O)OCC(CO)(CO)CO</smiles>

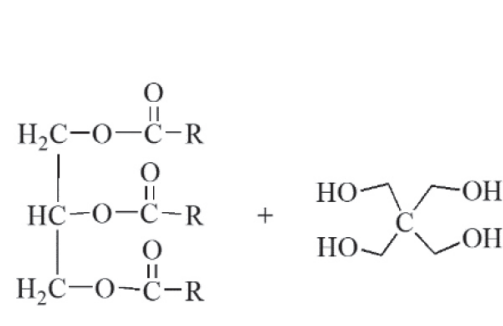

Aceite de higuerilla

Pentaeritritol

$\mathrm{R}=\left(\mathrm{H}_{2} \mathrm{C}\right)_{7}-\underset{\mathrm{H}}{\mathrm{C}}=\stackrel{\mathrm{H}}{\mathrm{C}}-\stackrel{\mathrm{C}}{\mathrm{C}} \stackrel{\stackrel{\mathrm{OH}}{\mathrm{I}}}{\mathrm{C}}-\left(\mathrm{CH}_{2}\right)_{5}-\mathrm{CH}_{3}$<smiles>[R]C(=O)OCC(O)CO</smiles><smiles>[R]C(=O)OC(CO)CO</smiles>

(b)<smiles>O=NCCCCCCCNC(=O)O</smiles>

1,6-diisocianato de hexametileno<smiles>CC1(CN=O)CCC(C)(C)C([N+](=O)[O-])C1</smiles>

Diisocianato de isoforona

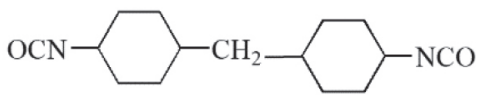

Figura 1. (a) Esquema de modificación química del poliol (b) Estructura química de diisocianatos empleados en la síntesis de PUs

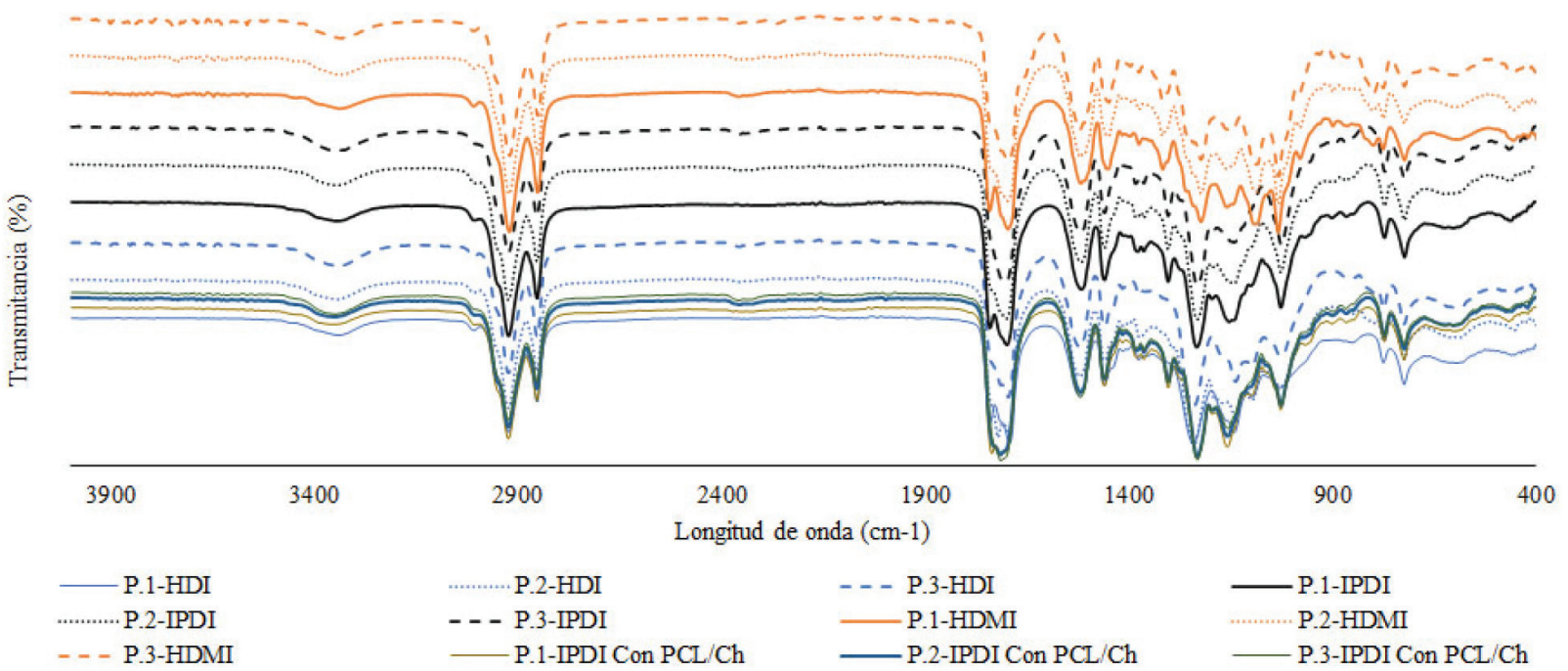

Figura 2. Espectros de FTIR de PUs sintetizados según el tipo de poliol y diisocianato

campo de la ingeniería tisular. Los valores de densidad reportados por los autores oscilan entre 1,13 y $1,14 \mathrm{~g} \mathrm{~mL}^{-1} \cdot{ }^{24}$ Con lo anterior se puede inferir que los PUs sintetizados se pueden emplear para el diseño de biomateriales.

Las altas densidades de materiales poliméricos se pueden relacionar con un mayor contenido de hidroxilo $(\mathrm{OH})$ debido al aumento de las reacciones de reticulación. ${ }^{28}$ En la investigación realizada por Carriço y col. observaron que al aumentar el contenido de aceite de higuerilla en la formulación de espuma se incrementaba la densidad aparente, lo que sugiere que las cadenas de polímero estaban más empaquetadas con menor volumen libre y células más pequeñas, lo que a su vez aumentaba la rigidez de estos materiales. ${ }^{28}$

\section{Propiedades mecánicas}

A las matrices de PUs sintetizadas (18 láminas) se les determinaron las propiedades mecánicas (resistencia a la tensión, porcentaje de elongación y módulo de Young) por medio de la determinación de las curvas de tensión-deformación. En la Figura 4 


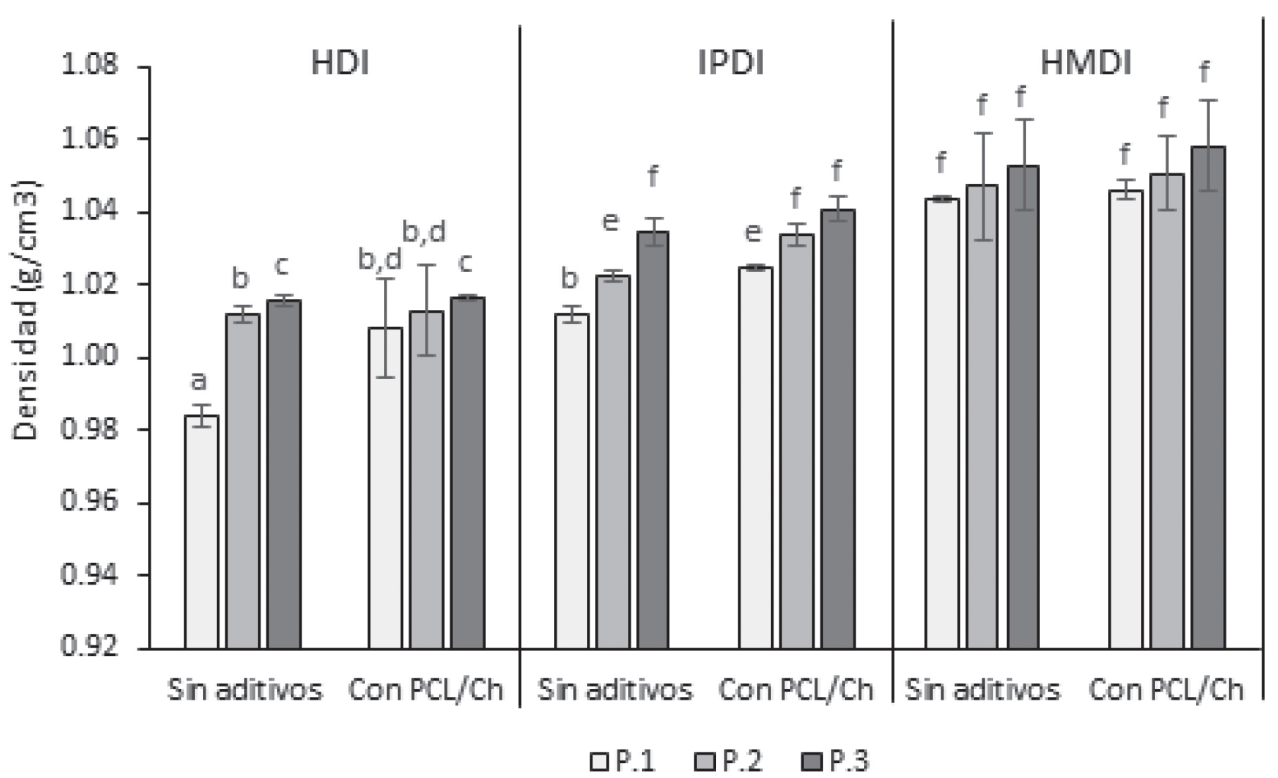

Figura 3. Densidad de los PUs sintetizados con IPDI para cada poliol empleado. Los datos están presentados como la media $\pm S D$ (n=3). Barras con letras diferentes ( $a . b, c, d, e, f)$ indican diferencias significativas $(p<0,05)$

se presentan los resultados de la resistencia a la tensión por medio del esfuerzo máximo, el porcentaje de elongación, el módulo de Young de las matrices sintetizadas según el tipo de poliol, el diisocianato empleado en la síntesis y los aditivos utilizados (PCL/Ch) y las curvas de tensión-deformación de los materiales.

Los materiales empleados en la síntesis, como los isocianatos, tienen una fuerte influencia sobre las propiedades físicas y mecánicas, tales como el módulo de Young, resistencia a la tracción o flexibilidad, debido a la estructura celular y molecular de los mismos. ${ }^{3}$ Esto se da porque la naturaleza de los enlaces y las sub-unidades de cada estructura tienen un efecto sobre las propiedades mecánicas. ${ }^{29}$ Es así como en la Figura 4 se observa que la estructura del diisocianato utilizado influye en las propiedades mecánicas presentando diferencias estadísticas significativas $(\mathrm{p}<0,05)$. Se observa que cadenas lineales como la del HDI genera PUs con menores propiedades mecánicas (esfuerzo máximo y porcentaje de elongación) comparado con las estructuras cicloalifáticas que contienen anillos en las estructuras como son el IPDI y el HMDI.

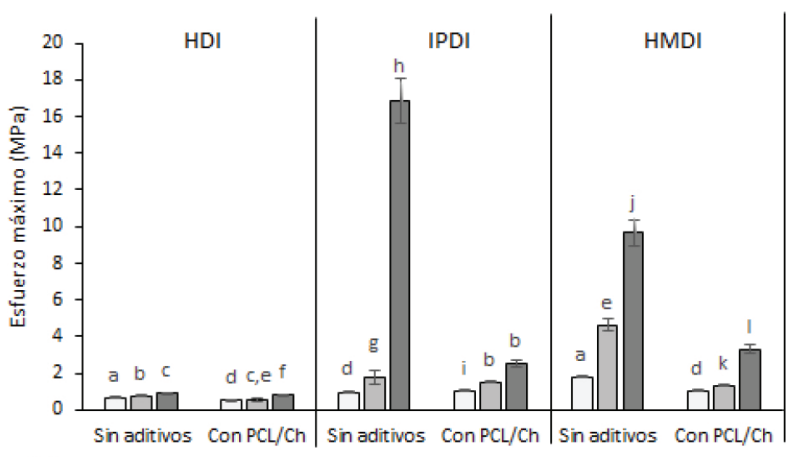

(a)

\begin{tabular}{lll} 
口P.1 & 口P.2 \\
\hline$P .3$
\end{tabular}

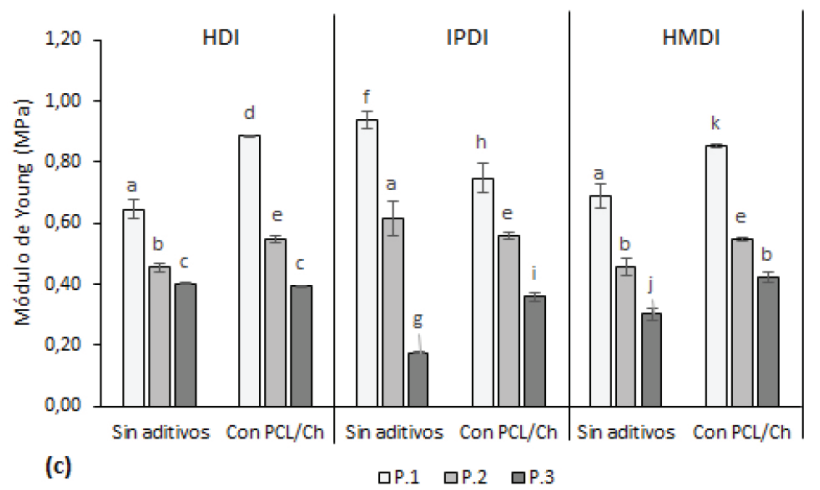

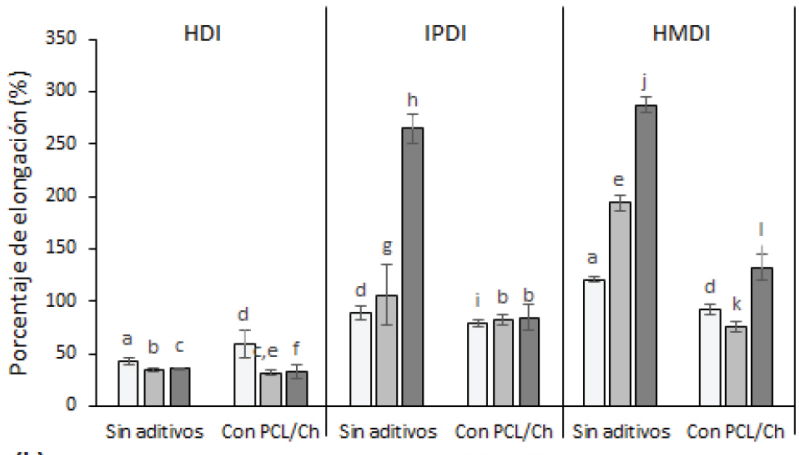

(b)

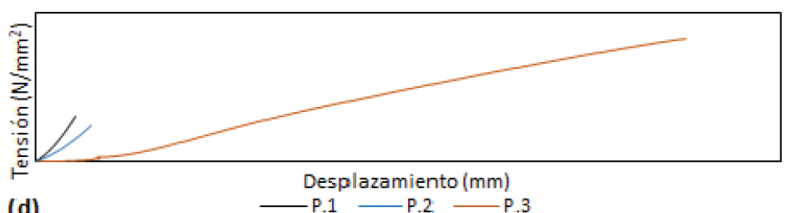

(d)

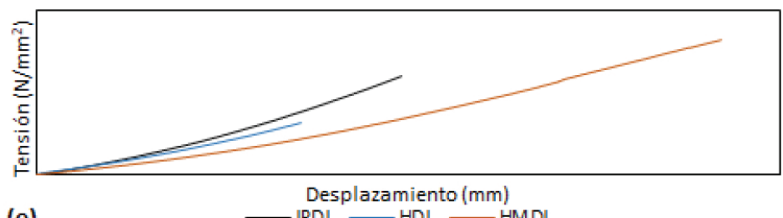

(e)

Figura 4. Propiedades mecánicas de PUs sintetizados según el tipo de poliol y diisocianato. (a) Esfuerzo máximo de PUs. (b) Porcentaje de elongación de PUs. (c) Módulo de Young de PUs. (d) Curva de tensión-deformación del diisocianato IPDI con los polioles P.1, P.2 y P.2. (e) Curva de tensión-deformación del poliol P.1 con los diisociantos HDI, IPDI y HMDI. Los datos están presentados como la media $\pm S D(n=3)$. Barras con letras diferentes (a. $b, c, d$, e, $f$, $g$, $h, i, j, k, l)$ indican diferencias significativas $(p<0,05)$ con respecto al tipo de poliol 
Algunos de los factores que definen el comportamiento reológico de los materiales sintetizados dependen de la estructura química de los diisocianatos empleados, entre los que se puede mencionar la isomería conformacional, los enlaces, el tamaño molecular, entre otros. ${ }^{30}$ Como se observa en la Figura 4 la geometría de los diisocianatos (alifáticos, cicloalifáticos) influye sobre las propiedades mecánicas como el esfuerzo máximo y el porcentaje de elongación de los PUs. ${ }^{8}$ Es importante mencionar que el HDI consiste en una cadena lineal entre los dos grupos isocianato, el IPDI y el HMDI son diisocianatos cicloalifáticos que contienen uno y dos anillos respetivamente, entre los dos grupos isocianato. Es por esto que se puede inferir que el tamaño de la molécula y el impedimento estérico que presentan los diisocianatos alifáticos cíclicos (cicloalifáticos), favorece la densidad de entrecruzamiento, ${ }^{30}$ dado que la geometría afecta directamente los puentes de hidrógeno entre los grupos uretano mejorando las propiedades mecánicas. ${ }^{8}$

En la Figura 4 (a) se observa que el mayor valor de esfuerzo máximo (16,86 MPa) se obtuvo con el diisocianato IPDI y el poliol P.3. Esto pudo suceder por los tres grupos metilo $\left(-\mathrm{CH}_{3}\right)$ presentes en la estructura del IPDI. ${ }^{8}$ Adicional a esto, la estructura del IPDI que es no lineal y no coplanar conduce a un menor empaquetamiento y pocos dominios duros lo que aumenta el nivel segmentario molecular de las cadenas del PU. ${ }^{3}$

En una investigación desarrollada por Javaid y col. sintetizaron PUs con tres diisocianatos diferentes, HDI (alifático), IPDI (cicloalifático) y diisocianato de tolueno (TDI) (aromático) con Ch y PCL, y evaluaron el efecto de la geometría del diisocianato sobre las propiedades mecánicas del material. Los autores encontraron que el TDI presentó mayor resistencia a la tracción por el carácter aromático del diisocianato, y el resultado más bajo corresponde a las matrices sintetizadas con HDI por la baja reactividad que tiene este diisocianato relacionada con la estructura alifática lineal que tiene. ${ }^{8}$

En cuanto al tipo de poliol (derivados del aceite de higuerilla: P.1, P.2 y P.3) se observa en la Figura 4(a) un incremento en el esfuerzo máximo de todos los materiales sintetizados con el poliol P.3 y los diisocianatos IPDI y HMDI. El poliol P. 3 es el que presenta mayor grado de entrecruzamiento físico dados por el incremento de grupos hidroxilo en la cadena del poliol al modificarlo con pentaeritritol. ${ }^{26}$ Es así como, un incremento en el índice hidroxilo de los polioles incrementa los sitios reactivos disponibles para los enlaces NCO de los diisocianatos, con lo que se obtiene un incremento de la densidad de entrecruzamiento del PU y un material con estructura más compacta. ${ }^{14}$ Adicionalmente, las interacciones de los puentes de hidrógeno generan una fuerte atracción entre los dominios de segmentos blandos y duros quienes definen la rigidez y estabilidad de los polímeros. ${ }^{9,31}$

En la Figura 4(b) se presentan los resultados relacionados con el porcentaje de elongación a la ruptura y se observa un incremento del porcentaje a medida que se incrementa el grado de modificación del poliol con diferencias estadísticas significativas $(\mathrm{p}<0,05)$ del poliol P. 1 respecto de los polioles P.2 y P.3. Es probable que la flexibilidad de los materiales esté dada por la larga cadena de hidrocarburos del aceite presentes en la cadena del polímero. ${ }^{32}$ También se observó un efecto por el tipo de diisocianato, ya que los valores más altos se lograron con los diisocianatos alifáticos cíclicos. De acuerdo con lo mencionado en los párrafos anteriores, las propiedades mecánicas de los PUs dependen tanto de la estructura de los monómeros como de la densidad de entrecruzamiento y el contenido de puentes de hidrógeno intermolecular. ${ }^{32,33}$

Al analizar el comportamiento de los aditivos empleados en la síntesis sobre las propiedades mecánicas (15\% de PCL y $3 \%$ de $\mathrm{Ch}$ ), se puede observar (Figura 4(a)) que para el diisocianato HDI no se afecta el esfuerzo máximo con la adición de PCL/Ch. En el caso del diisocianato IPDI se encuentran diferencias estadísticas cuando se utilizan aditivos (PCL/Ch) con los tres polioles (P.1, P.2 y P.3), observando una disminución de las propiedades mecánicas. Por último, para el diisocianato HMDI se observa que para P.1, P.2 y P.3 se disminuye el esfuerzo máximo al adicionar PCL/Ch con respecto a control.

Un efecto similar se observa para el porcentaje de elongación (Figura 4(b)), ya que para el IPDI y HMDI se disminuye el porcentaje de elongación cuando se adiciona PCL/Ch con el poliol P.3. El resto de los materiales no presentan diferencias significativas con los aditivos empelados respecto al control.

En cuanto al módulo de Young en la Figura 4(c) se observa que el material que presenta el valor más alto del módulo de Young corresponde al sintetizado con el diisocianato IPDI para el poliol P.1 con un valor de $0.937 \pm 0.029 \mathrm{MPa}$. El comportamiento de los polioles P.1, P.2 y P.3 es similar para los tres diisocianatos donde se observa que a medida que se incrementan los grupos hidroxilo el módulo tiende a disminuir. La adición de PCL/Ch tiende a incrementar el módulo para los diisocianatos HDI y HMDI. En el caso del IPDI se observa que los aditivos disminuyen el módulo de Young. De acuerdo con los resultados obtenidos del módulo de Young, estos materiales se podrían utilizar para el diseño de polímeros flexibles. ${ }^{34}$ Materiales flexibles son aptos para el diseño de biomateriales como tejidos blandos, tejidos del músculo esquelético, entre otros. ${ }^{26,34}$ Así, por ejemplo, materiales con módulos de elasticidad superiores a $10 \mathrm{kPa}$ se utilizan para diseñar estructuras a escalas micro que mimetizan tejidos vivos, ${ }^{25} \mathrm{y}$ el módulo elástico de tejidos como la piel humana presenta valores entre $50 \mathrm{kPa}$ y $100 \mathrm{MPa} .^{35,36}$ Por lo anterior, se puede inferir que los materiales sintetizados en esta investigación pueden ser aptos para el diseño de biomateriales por las propiedades mecánicas obtenidas.

Adicional a esto se presentan las curvas de tensión-deformación en la Figura 4(d) y 4(e) para el diisocianato IPDI con los tres polioles (P.1, P.2 y P.3) y para el poliol P.1 con los tres diisocianatos (HDI, IPDI y HMDI), respectivamente. En estas figuras se puede observar el comportamiento que ya se ha descrito en los párrafos anteriores donde el poliol con el mayor índice hidroxilo presenta los resultados más elevados de las propiedades mecánicas. De igual forma, la estructura del diisocianato tiene un efecto significativo sobre las propiedades mecánicas de los materiales sintetizados.

\section{Propiedades térmicas}

Análisis termogravimétrico (TGA). El análisis termogravimétrico se realizó por medio de termogramas de los PUs. En la Figura 5 se presentan los resultados para las matrices sintetizadas según el tipo de poliol y el diisocianato.

Según se presenta en la Figura 5 para los PUs sintetizados con los tres polioles y los tres diisocianatos, se conserva una tendencia con respecto al comportamiento térmico, lo que indica que no se presentan desplazamientos de las temperaturas de degradación con el uso de polioles modificados ni con la variación del diisocianato. Al obtener las curvas derivadas del análisis termogravimétrico se observó la presencia de varios picos, lo que concuerda con otros autores que afirman que el mecanismo de degradación de los PUs es complejo por la formación de diversos compuestos en el proceso. ${ }^{10,37}$

Para cada una de las matrices poliméricas se obtuvo un termograma, con el cual se determinó la estabilidad de los PUs sintetizados en este estudio (los resultados completos no se muestran). Se observó que el tipo de poliol, diisocianato y aditivos no afectan la estabilidad térmica de los materiales al compararlo con el material sintetizado con poliol y diisocianato sin aditivos. Con las curvas de degradación se determinó que la estabilidad de los PUs se presentaba a temperaturas inferiores a $300{ }^{\circ} \mathrm{C}$ y una temperatura de 
(a)

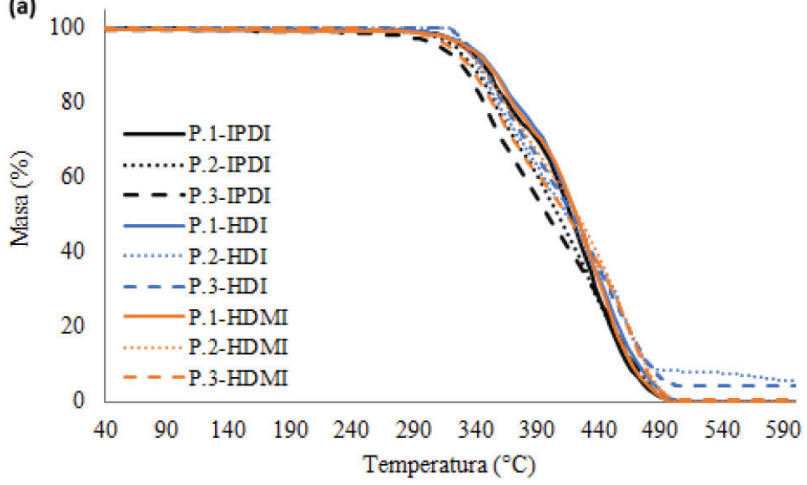

(b)

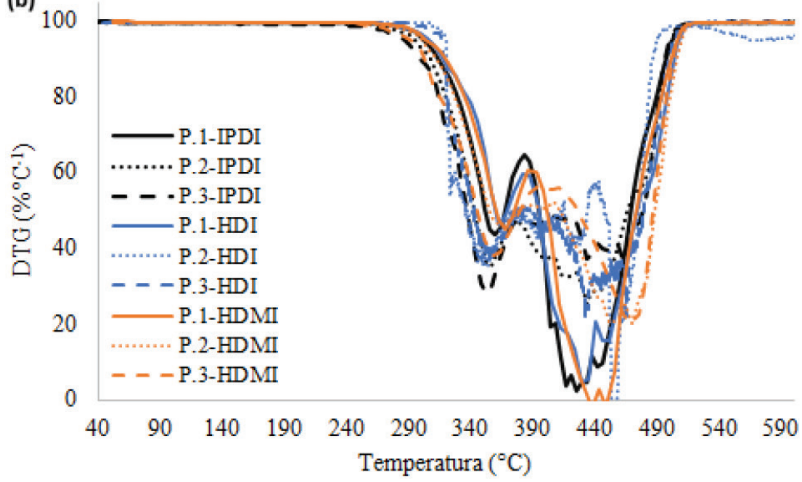

Figura 5. Termogramas de los PUs sintetizados según el tipo de poliol y diisocianato. (a) Curva TG de los PUs. (b) Curva DTG de los PUs

degradación completa de los materiales a $600{ }^{\circ} \mathrm{C}$ lo que concuerda con el comportamiento típico de materiales tipo PU. ${ }^{9}$

Con los termogramas se pudo determinar diferentes etapas de degradación que presentan los PUs. Una etapa está alrededor de 250$370{ }^{\circ} \mathrm{C}$, otra entre $370-430{ }^{\circ} \mathrm{C}$ y una última etapa cerca de $420{ }^{\circ} \mathrm{C}$. En la primera etapa se puede dar la degradación de enlaces uretano, la segunda etapa puede estar relacionada con la degradación térmica de los segmentos suaves, y la última etapa se encuentra en un rango de $425-500{ }^{\circ} \mathrm{C}$ y corresponde a la degradación térmica de grupos -OH remanentes del poliol. ${ }^{9,10,38}$

\section{Calorimetría diferencial de barrido (DSC).}

En la Figura 6 se presentan las curvas DSC de los materiales sintetizados con el diisocianato IPDI con cada uno de los polioles (p.1, P.2 y P.3) y en la Tabla 1 se encuentran los resultados de la temperatura de transición vítrea $\left(T_{g}\right)$ de los PUs sintetizados. Estos valores se determinaron de la evaluación de las curvas de DSC de los materiales.

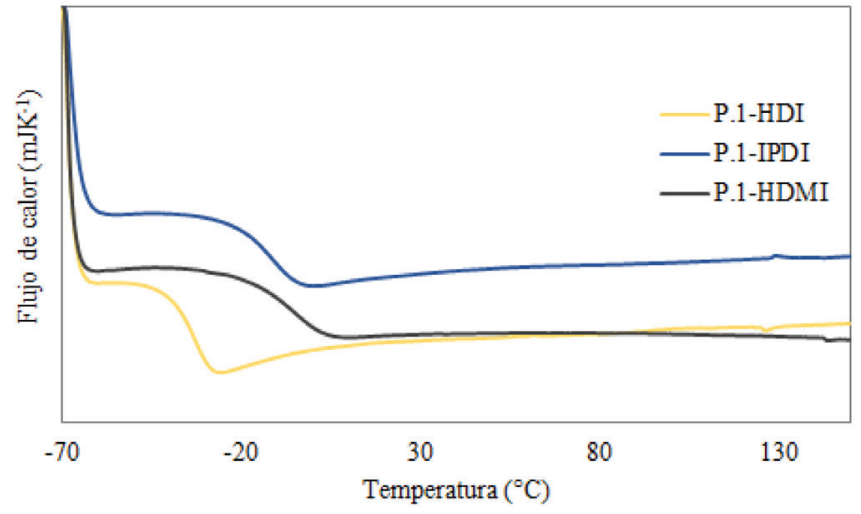

Figura 6. Termogramas DSC de PUs sintetizados con el poliol P.1 y con diferente diisocianato

Tabla 1. Temperatura de transición vítrea (Tg) de los PUs sintetizados

\begin{tabular}{lcccc}
\hline \multirow{2}{*}{ Diisocianato } & \multirow{2}{*}{ Aditivos } & \multicolumn{3}{c}{$\mathrm{Tg}\left({ }^{\circ} \mathrm{C}\right)$} \\
\cline { 3 - 5 } & & $\mathrm{P} .1$ & $\mathrm{P} .2$ & $\mathrm{P} .3$ \\
\hline \multirow{2}{*}{ HDI } & Sin aditivos & -35.82 & -24.17 & -23.05 \\
& Con PCL/Ch & -40.16 & -23.13 & -31.35 \\
\hline \multirow{2}{*}{ IPDI } & Sin aditivos & -14.80 & -12.80 & 14.80 \\
& Con PCL/Ch & -25.40 & -14.80 & 2.80 \\
\hline \multirow{2}{*}{ HMDI } & Sin aditivos & -8.57 & -6.71 & 5.43 \\
& Con PCL/Ch & -20.21 & -11.4 & 3.42 \\
\hline
\end{tabular}

En la Figura 6 se observa el comportamiento de los materiales en cuanto al tipo de diisocianato utilizado en la síntesis para un mismo poliol (P.1). A medida que el diisocianato presenta un anillo en la estructura, la $T_{g}$ tiende a incrementar. Lo mismo se presenta en la Tabla 1 donde se observa que el tipo de diisocianato influye significativamente sobre la $T_{g}$. Esto coincide con lo explicado en las propiedades mecánicas sobre la estructura molecular del diisocianato. Los valores de la $T_{g}$ del poliol P.1 con los diisocianatos HDI, IPDI y HMDI sin aditivos fueron $-35,82{ }^{\circ} \mathrm{C},-14,80{ }^{\circ} \mathrm{C}$ y $-8,57^{\circ} \mathrm{C}$, respectivamente. Es así como el diisocianato alifático lineal (HDI) presentó los valores más bajos de $T_{g}$ (entre $-40{ }^{\circ} \mathrm{C}$ y $-20{ }^{\circ} \mathrm{C}$ aproximadamente). Los diisocianatos alifáticos con anillos como el IPDI y el HMDI presentaron valores superiores de $T_{g}$, posiblemente debido a la energía necesaria para reorganizar las moléculas del PU. La $T_{g}$ es una propiedad que está relacionada con el volumen libre molecular y los movimientos segmentarios de las cadenas. ${ }^{3}$

PUs sintetizados con HDI y poli(adipato de etilenglicol) macroscópicamente homogéneo y con alta flexibilidad presentó un valor de $T_{g}$ de $-43{ }^{\circ} \mathrm{C}$ correspondientes a los segmentos suaves derivados de las cadenas largas del poliol utilizado. ${ }^{4}$

De igual forma, se observa que el tipo de poliol también presenta un efecto sobre la $T_{g}$, es así como la tendencia observada consiste en disminuir la $T_{g}$ a medida que se disminuye el índice hidroxilo, lo que coincide con el tipo de entrecruzamiento de los PUs sintetizados, indicando que se necesita una energía mayor para reordenar la estructura por un cambio entre las fuerzas intermoleculares. Esto puede ser debido a interacciones secundarias resultantes de la estructura hiperramificada generada. ${ }^{39}$ El triglicérido del ácido ricinoléico del aceite de higuerilla empleado como poliol contiene una estructura ordenada donde los grupos hidroxilo tienen una distribución uniforme dentro de la cadena, lo que permite obtener un PU con una estructura uniformemente reticulada con lo que se logran propiedades mecánicas elevadas y estabilidad térmica. ${ }^{40}$ Las propiedades térmicas de los PUs dependen de la cantidad de enlaces uretano presentes en la estructura, ya que éstos pueden tolerar una cantidad considerable de calor. ${ }^{41}$ Es así como se observa que el incremento de la $T_{g}$ pueda estar relacionado con el incremento de los segmentos duros en el PU lo que afecta de forma directa la movilidad de las cadenas. ${ }^{42}$

La adición de PCL/Ch tiende a disminuir el valor de la $T_{g}$ de los materiales comparado con las matrices sin aditivos a las concentraciones estudiadas, lo que indicaría que se favorecería la interacción entre los aditivos y los segmentos cristalinos y se presentaría una baja interacción entre los aditivos y los segmentos amorfos, de acuerdo con lo descrito por Anbinder y col. ${ }^{43}$ Con lo expuesto en los párrafos anteriores, teniendo en cuenta los resultados de las propiedades sobre los PUs sintetizados, se puede inferir que los PUs son estables térmicamente y que pueden ser empleados para el diseño de biomateriales. 


\section{Ángulo de contacto}

En la Figura 7 se presentan los resultados del ángulo de contacto de los PUs sintetizados.

Según se observa en la Figura 7 el poliol P.1 no presenta diferencias significativas con los diisocianatos empleados. Caso similar sucede con P.2 para los diisocianatos HDI e IPDI, puesto que para HMDI se presenta una disminución del ángulo de contacto. Para P.3 se observan diferencias estadísticas con los tres diisocianatos debido a que se presenta una reducción de los valores del ángulo con el diisocianato lineal, indicando una disminución del carácter hidrofóbico. Esto probablemente sea debido a la rigidez del material, lo que a su vez incrementa o disminuye la afinidad por el agua. Lo anterior se infiere teniendo en cuenta que el ángulo de contacto puede reflejar de forma indirecta los cambios en la estructura de la superficie y la composición del material. ${ }^{44}$ Adicional a esto, una estructura que presente mayor entrecruzamiento, promueve la formación de una capa protectora en la superficie lo que repele la intrusión del agua. ${ }^{44}$
Según el análisis estadístico se puede inferir que la adición de PCL/Ch sobre los valores del ángulo de contacto, no presenta diferencias significativas $(\mathrm{p}<0,05)$ y por lo tanto no influyen sobre el carácter hidrofílico. Se encontraron valores cercanos a 100 grados, por lo que se puede deducir que los materiales tienden a ser de carácter hidrofóbico y se pueden emplear para el diseño de biomateriales.

\section{Ensayo in vitro de viabilidad celular}

Como parte de la evaluación biológica in vitro de los PUs se determinó el porcentaje de viabilidad celular sobre una línea celular de fibroblastos de ratón L-929 y los resultados se muestran en la Figura 8.

En la Figura 8 se observa que todos los polímeros sintetizados presentan viabilidad celular superior al $70 \%$ con lo que se puede inferir que todos los PUs se pueden utilizar como materiales para el diseño de biomateriales según lo estipula la norma ISO/CD 10993-5. De acuerdo con los resultados se observa que los valores más altos de viabilidad celular (superior al 100\%) corresponden al diisocianato

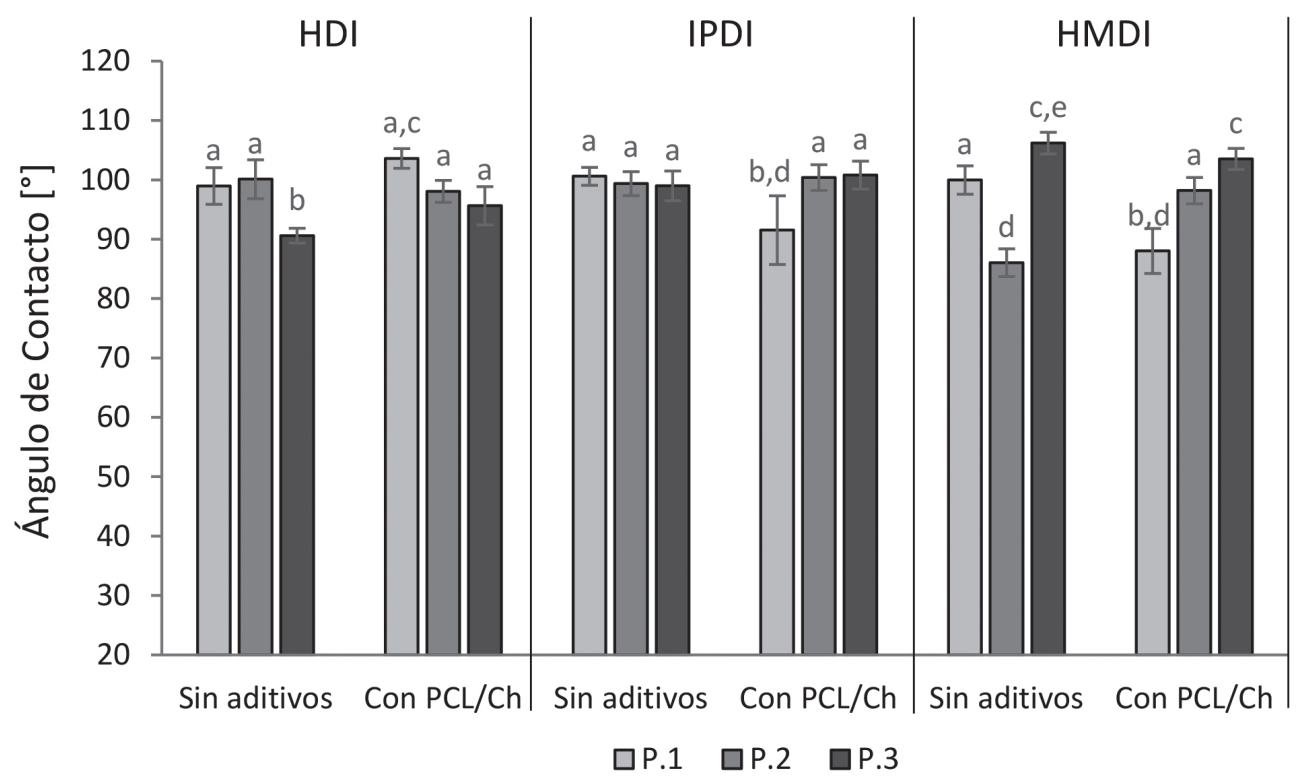

Figura 7. Ángulo de contacto de PUs sintetizados según el tipo de poliol y diisocianato. Los datos están presentados como la media $\pm S D(n=10)$. Las barras con letras diferentes $(a . b, c, d, e)$ indican diferencias significativas $(p<0,05)$

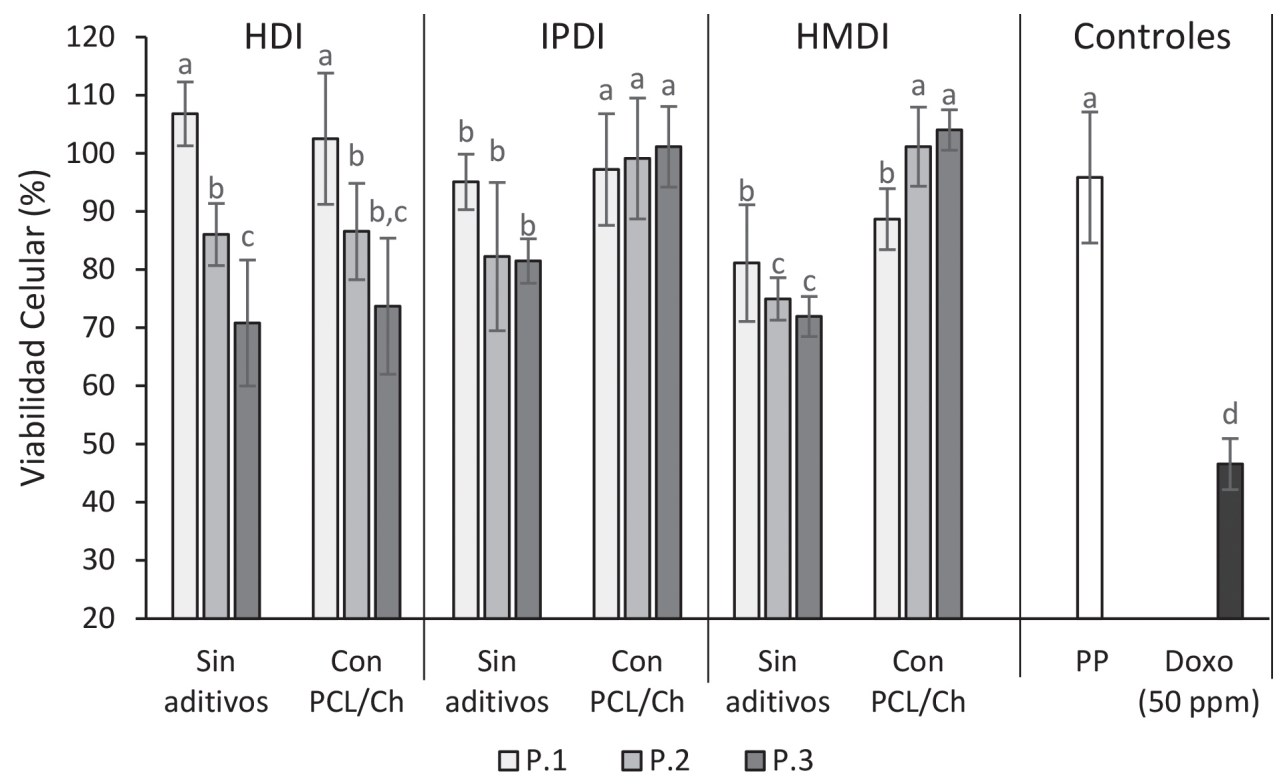

Figura 8. Porcentaje de viabilidad celular a 24 h sobre Fibroblastos de ratón L-929. Los datos están presentados como la media $\pm S D$ ( $n=3$ ). Barras con letras diferentes $(a . b, c, d)$ indican diferencias significativas $(p<0,05)$ entre los polioles 
alifático lineal, el HDI. Adicional a esto, al analizar los materiales sin aditivos y con el poliol sin modificación (P.1) se observa que el porcentaje de viabilidad celular tiende a disminuir cuando el diisocianato utilizado contiene anillos en la estructura (IPDI y HMDI). Al observar el efecto que presenta el tipo de poliol sobre la viabilidad celular se puede decir que no se presentan diferencias estadísticas $(\mathrm{p}<0.05)$, pero la tendencia es que a medida que se incrementa el entrecruzamiento de los materiales la viabilidad disminuye. Es probable que el elevado grado de entrecruzamiento genere una baja disponibilidad de grupos funcionales, como grupos - $\mathrm{OH}$, en el polímero entrecruzado, lo que podría disminuir la adhesión celular. ${ }^{26}$

Un biomaterial a base de polipropileno (PP) fue usado como control para comparar los resultados de viabilidad de los PUs con un material comercialmente disponible. Como se observa en la Figura 8, la viabilidad celular del $60 \%$ de los PUs sintetizados no presentan diferencias estadísticas $(\mathrm{p}<0.05)$ comparado con el PP. Adicional a esto, se utilizó un material como control negativo (Doxo) y se observa que ninguno de los materiales sintetizados presenta toxicidad sobre las células de fibroblastos de ratón L-929.

De acuerdo con los resultados sobre el porcentaje de viabilidad celular se puede inferir que los PUs sintetizados son aptos como posibles biomateriales. La tendencia que se observa para los fibroblastos L-929 es que a medida que se incrementa el grado de entrecruzamiento del PU, se disminuye el porcentaje de viabilidad celular.

El objetivo de adicionar la mezcla de PCL/Ch era modificar la biocompatibilidad de las matrices de PU sintetizadas dado que tanto la PCL como el Ch poseen elevada biocompatibilidad y biodegradabilidad que los hace interesantes para las aplicaciones biomédicas. ${ }^{8}$ Se ha demostrado que PUs sintetizados con diisocianatos aromáticos como el TDI, se caracterizan por presentar una baja hemocompatibilidad, son mutagénicos y citotóxicos, pero al utilizar polímeros como el $\mathrm{Ch}$ se encontraron PUs con un buen comportamiento biológico. ${ }^{45}$ De acuerdo con los resultados de la Figura 8 se observa que el uso de los aditivos (PCL/Ch) incrementa los porcentajes de viabilidad celular cuando se utilizan diisocianatos cicloalifáticos y polioles modificados químicamente.

Con fibroblastos como NIH/3T3 se evaluó la adhesión celular sobre películas e injertos de PU a base de policaprolactona, bis(ciclohexil-isocianato) de 4,4'-metileno PCL:HMDI:lysine L-arginina durante un período de tres días y se observó una buena adhesión de los fibroblastos sobre los materiales. Las anteriores propiedades hacen que estos PUs sean aptos para aplicaciones cardiovasculares, principalmente injertos vasculares de pequeño diámetro. ${ }^{46}$

\section{CONCLUSIONES}

Al diseñar biomateriales como injertos cardiovasculares, venas, arterias, entre otros, es importante tener en cuenta el comportamiento y la función mecánica ya que las propiedades mecánicas de cada dispositivo son diferentes. Por lo tanto, la evaluación de las propiedades mecánicas de cada material polimérico sintetizado es fundamental para determinar las aplicaciones del material. Adicionalmente, es igual de importante determinar el comportamiento de los materiales sintetizados sobre alguna línea celular para determinar la viabilidad celular de los mismos. Es así como a los PUs sintetizados se les evaluaron las propiedades mecánicas y biológicas y se determinó que los PUs pueden tener potencial como materiales para el diseño de dispositivos biomédicos. Las propiedades mecánicas mostraron una fuerte influencia relacionada con el tipo de poliol utilizado ya que al modificar los grupos hidroxilo se pueden obtener cadenas de polímeros con mayor grado de entrecruzamiento. De igual forma se pudo determinar que el tipo de diisocianato afecta las propiedades mecánicas debido al efecto de la estructura molecular de cada diisocianato. Es así como los diisocianatos cicloalifáticos presentan un incremento en las propiedades mecánicas. Los valores del módulo de Young se encontraron dentro del rango óptimo para el diseño de biomateriales. El porcentaje de elongación, con un máximo de $287 \%$, indica que es posible obtener materiales resistentes con flexibilidad, lo que permite diseñar biomateriales que no causen lesiones a los tejidos suaves. Con los resultados de viabilidad celular se encontró que los materiales no son tóxicos para fibroblastos L-929 con lo que se podría esperar que dichos materiales puedan ser empleados como biomateriales sin generar efectos secundarios. Adicional a esto, la adición de polímeros de cadena corta como la PCL y el Ch mejoran las propiedades biológicas de los materiales. De acuerdo con los resultados se puede proponer a los PUs sintetizados con polioles derivados del aceite de higuerilla (Ricinus communis) y los diisocianatos IPDI, HDI y HMDI, con adición de PCL/Ch como candidatos para el diseño de biomateriales.

\section{AGRADECIMIENTOS}

A la Universidad de La Sabana por la financiación del proyecto de investigación ING-205-2018, a Colciencias por la beca-préstamo doctoral bajo la convocatoria 617-2 de 2014 por medio de la Gestión de Becas Externas (GBE) y a la Asociación Universitaria Iberoamericana de Postgrado (AUIP) por la beca de movilidad internacional.

\section{REFERENCIAS}

1. Xie, F.; Zhang, T.; Bryant, P.; Kurusingal, V.; Colwell, J. M.; Laycock, B.; Prog. Polym. Sci. 2019, 90, 211.

2. Sliozberg, Y. R.; Gair, J. L.; Hsieh, A. J.; Polymer (Guildf) 2020, 193, 122339.

3. Choe, H.; Kim, J. H.; J. Ind. Eng. Chem. 2019, 69, 153.

4. Beneš, H.; Vlčková, V.; Paruzel, A.; Trhlíková, O.; Chalupa, J.; Kanizsová, L.; Skleničková, K.; Halecký, M.; Polym. Degrad. Stab. 2020, 176, 1 .

5. Asensio, M.; Costa, V.; Nohales, A.; Bianchi, O.; Gómez, C. M.; Polymers (Basel) 2019, 11,

6. Ng, W. S.; Lee, C. S.; Chuah, C. H.; Cheng, S. F.; Ind. Crops Prod. 2017 $97,65$.

7. Ionescu, M.; Radojčić, D.; Wan, X.; Shrestha, M. L.; Petrović, Z. S.; Upshaw, T. A.; Eur. Polym. J. 2016, 84, 736.

8. Javaid, M.; Younas, M.; Zafar, I.; Ahmad, R.; Mahmood, K.; Jabeen, S.; Int. J. Biol. Macromol. 2019, 124, 321.

9. Jutrzenka Trzebiatowska, P.; Santamaria Echart, A.; Calvo Correas, T.; Eceiza, A.; Datta, J.; Prog. Org. Coatings 2018, 115, 41.

10. Venkatesh, D.; Jaisankar, V.; Mater. Today Proc. 2019, 14, 482.

11. Calvo-Correas, T.; Santamaria-Echart, A.; Saralegi, A.; Martin, L.; Valea, Á.; Corcuera, M. A.; Eceiza, A.; Eur. Polym. J. 2015, 70, 173.

12. Uscátegui, Y.; Arévalo, F.; Díaz, L.; Cobo, M.; Valero, M.; J. Biomater. Sci., Polym. Ed. 2016, 27, 1860.

13. Omonov, T. S.; Kharraz, E.; Curtis, J. M.; Ind. Crops Prod. 2017, 107, 378.

14. Moghadam, P.; Yarmohamadi, M.; Hasanzadeh, R.; Nuri, S.; Int. J. Adhes. Adhes. 2016, 68, 273.

15. Anirudhan, T. S.; Nair, S. S.; Nair, A. S.; Carbohydr. Polym. 2016, 152, 687.

16. Usman, A.; Zia, K. M.; Zuber, M.; Tabasum, S.; Rehman, S.; Zia, F.; Int. J. Biol. Macromol. 2016, 86, 630.

17. Wu, G.-H.; Hsu, S.; Colloids Surf., B 2016, 146, 825.

18. Wu, H.; Williams, G. R.; Wu, J.; Wu, J.; Niu, S.; Li, H.; Wang, H.; Zhu, L.; Carbohydr. Polym. 2018, 180, 304. 
19. Uscategui, Y. L.; Díaz, L. E.; Valero, M. F.; Quim. Nova 2017, 40, 131

20. Villegas-villalobos, S.; Díaz, L. E.; Vilariño-feltrer, G.; Vallés-Lluch, A.; Gómez-Tejedor, J. A.; Valero, M. F.; J. Mater. Res. 2018, 33, 2598

21. Valero, M. F.; Ortegón, Y.; J. Elastomers Plast. 2015, 47, 360.

22. Mekewi, M. A.; Ramadan, A. M.; ElDarse, F. M.; Abdel Rehim, M. H.; Mosa, N. A.; Ibrahim, M. A.; Egypt. J. Pet. 2017, 26, 9.

23. Shah, S. A. A.; Imran, M.; Lian, Q.; Shehzad, F. K.; Athir, N.; Zhang, J.; Cheng, J.; React. Funct. Polym. 2018, 128, 97.

24. Conejero-García, Á.; Gimeno, H. R.; Sáez, Y. M.; Vilariño-Feltrer, G.; Ortuño-Lizarán, I.; Vallés-Lluch, A.; Eur. Polym. J. 2017, 87, 406.

25. Rezvanain, M.; Ahmad, N.; Mohd Amin, M. C. I.; Ng, S. F.; Int. J. Biol. Macromol. 2017, 97, 131.

26. Vannozzi, L.; Ricotti, L.; Santaniello, T.; Terencio, T.; Oropesa-Nunez, R.; Canale, C.; Borghi, F.; Menciassi, A.; Lenardi, C.; Gerges, I.; J. Mech. Behav. Biomed. Mater. 2017, 75, 147.

27. Gregorí, B.; Guerra, M.; Mieres, G.; Alba, L.; Brown, A.; RangelVázquez, N.; Sosa, M.; de la Hoz, Y.; Revista Iberoamericana de Polímeros 2008, 9, 377.

28. Carriço, C. S.; Fraga, T.; Pasa, V. M. D.; Eur. Polym. J. 2016, 85, 53.

29. Temenoff, J. S.; Mikos, A. G.; Biomaterials Pearson/Prentice Hall: Upper Saddle River, 2008.

30. Borrero-López, A. M.; Valencia, C.; Franco, J. M.; Eur. Polym. J. 2017, $89,311$.

31. Braun, U.; Lorenz, E.; Weimann, C.; Sturm, H.; Karimov, I.; Ettl, J.; Meier, R.; Wohlgemuth, W. A.; Berger, H.; Wildgruber, M.; J. Mech. Behav. Biomed. Mater. 2016, 64, 281.

32. Thakur, S.; Karak, N.; Prog. Org. Coatings 2013, 76, 157.
33. Gurunathan, T.; Mohanty, S.; Nayak, S. K.; Prog. Org. Coatings 2015, $80,39$.

34. Park, H.; Gong, M.-S.; Knowles, J. C.; J. Mater. Sci. Mater. Med. 2013, 24, 281.

35. Wu, C.-S.; Int. J. Polym. Mater. Polym. Biomater. 2016, 65, 872.

36. Garg, B.; Sandhu, V.; Sood, N.; Sood, A.; Malhotra, V.; Pol. J. Pathol. 2012, 63, 172.

37. Cakić, S. M.; Ristić, I. S.; Cincović, M. M.; Nikolić, N. Č.; Nikolić, L.; Cvetinov, M. J.; Prog. Org. Coatings 2017, 105, 111.

38. Bakhshi, H.; Yeganeh, H.; Yari, A.; Nezhad, S. K.; J. Mater. Sci. 2014, 49, 5365.

39. Saikia, A.; Karak, N.; Polym. Degrad. Stab. 2017, 135, 8.

40. Zhang, C.; Garrison, T. F.; Madbouly, S. A.; Kessler, M. R.; Prog. Polym. Sci. 2017, 71, 91 .

41. Thakur, S.; Hu, J. Em Polyurethane; Yilmaz, F., org.; InTechOpen, 2017.

42. Sáenz-Pérez, M.; Lizundia, E.; Laza, J. M.; García-Barrasa, J.; Vilas, J. L.; León, L. M.; RSC Adv. 2016, 6, 69094.

43. Anbinder, P. S.; Peruzzo, P. J.; Amalvy, J. I.; Prog. Org. Coatings 2016, 101, 207.

44. Xu, C. an; Qu, Z.; Tan, Z.; Nan, B.; Meng, H.; Wu, K.; Shi, J.; Lu, M.; Liang, L.; Polym. Test. 2020, 86, 106485.

45. Javaid, M.; Zia, K.; Khera, R.; Jabeen, S.; Mumtaz, I.; Younis, M.; Shoaib, M.; Bhatti, I.; Int. J. Biol. Macromol. 2019, 129, 116.

46. Castillo-Cruz, O.; Avilés, F.; Vargas-Coronado, R.; Cauich-Rodríguez, J. V; Chan-Chan, L. H.; Sessini, V.; Peponi, L.; Mater. Sci. Eng. C 2019, $102,887$. 